\title{
A robust spectral method for solving Heston's model
}

\author{
E. Ngounda, K.C. Patidar and E. Pindza
}

\begin{abstract}
In this paper, we consider the Heston's volatility model (Heston in Rev. Financ. Stud. 6: 327-343, 1993]. We simulate this model using a combination of the spectral collocation method and the Laplace transforms method. To approximate the two dimensional PDE, we construct a grid which is the tensor product of the two grids, each of which is based on the Chebyshev points in the two spacial directions. The resulting semi-discrete problem is then solved by applying the Laplace transform method based on Talbot's idea of deformation of the contour integral (Talbot in IMA J. Appl. Math. 23(1): 97-120, 1979).
\end{abstract}

\section{Introduction}

The Heston's model is one of the most popular stochastic volatility models for derivative pricing. The model leads to a more realistic option price evaluation than the celebrated Black-Scholes model and constitutes its extension to the two-dimensional form $[1,2]$. In [3], Heston derived a semi-closed formula for the model; however, its implementation is not a straightforward exercise because of the oscillatory behaviour of the complex integrand which comes into play through the Fourier-type inversion formula. Therefore, we turn to numerical methods to approximate these option pricing problems.

Most of numerical methods for evaluating the Heston's model are based on the method-ofline approach which consists of two steps. Firstly, the PDE is discretized in space, thus generating a system of ordinary differential equations. Secondly, the subsequent semidiscrete problem is solved in time by applying a suitable time integration method. This is the approach followed in some of the numerical methods we review below.

In't Hout and Foulon [4] used the method-of-line approach to solve the Heston's model. They first discretized the PDE using a non-uniform grid to capture the important region around the strike price. Then they integrated the resulting semi-discrete problem by using four different alternating direction implicit (ADI) methods in time. As ADI methods, they considered the Douglas [5, 6], Craig-Sneyd [7], the modified Craig-Sneyd [7, 8] and the Hundsdorfer-Verwer $[9,10]$ schemes. Their approach was latter extended to the more complex Heston-Hull-White PDE in [11]. 
In [12], Ikonen and Toivanen studied the accuracy of the operator splitting methods for pricing American options with stochastic volatility. In their numerical experiments, they compared the accuracy of their approach with conventional implicit projective successive over relaxation (PSOR) time discretization method $[13,14]$. Their results demonstrated that the additional error, due to the splitting, does not increase the time discretization error.

Zhu and Chen [15] applied a singular perturbation method to price a European put option with a stochastic volatility model, and derived a simple analytical formula as an approximation for the valuation of European put options. In't Hout and Weideman [16] used finite differences to semi-discretize the Heston model in space and subsequently used the contour integral method for time integration. They compared the efficiency of the contour integral approach with the ADI splitting schemes for solving this problem. The numerical experiments showed that the contour integral method was superior for the range of medium to high accuracy requirements.

Some other interesting works dealing with volatility models and their applications can be found in [17-25] and some of the references therein.

In this paper, we apply the spectral method based on Chebyshev points to discretize the PDE in each spatial direction. Then we use a tensor product of one-dimensional polynomials to represent the two-dimensional basis functions. For the time discretization, we consider the contour integral methods. The rest of this paper is organized as follows. In Sect. 2, we discuss the Heston's model. In Sect. 3, we discuss the application of spectral methods to solve the model problem. Section 4 deals with the contour integral method for time discretization. Numerical results of our experiments are presented in Sect. 5. Finally, some concluding remarks and scope for future research are given in Sect. 6.

\section{Description of Model Problem}

Let $V \equiv V(s, \phi, \tau)$ denote the price of a European option described by the Hes- ton's stochastic volatility model at time $\tau=T-t$. Then, $V$ satisfies the following ton's PDE

$$
\begin{aligned}
\frac{\partial V}{\partial \tau}= & \frac{1}{2} s^{2} \varphi \frac{\partial^{2} V}{\partial s^{2}}+\rho \sigma s \varphi \frac{\partial V^{2}}{\partial s \partial \varphi}+\frac{1}{2} \sigma^{2} \varphi \frac{\partial^{2} V}{\partial \varphi^{2}}+r s \frac{\partial V}{\partial s} \\
& +\kappa(\varsigma-\varphi) \frac{\partial V}{\partial \varphi}-r V,
\end{aligned}
$$

where $0 \leq \tau \leq T, s>0$ and $\phi>0$. The parameter $\kappa>0$ is the mean-reversion rate, $\varsigma>0$ is the long-term mean, $\sigma>0$ is the volatility-of-variance, $\rho \in[-1,1]$ is the correlation between the two underlying Brownian motion, $r$ denotes the risk-free interest rate.

For a European put option, the payoff yields the initial condition 


$$
V(s, \varphi, 0)=\max (K-s, 0),
$$

where $K>0$ is the strike price of the option. In numerical practice, a bounded spatial domain $\left[0, s_{\max }\right] \times\left[0, \phi_{\max }\right]$ is chosen with fixed values $s_{\max }, \phi_{\max }$ taken sufficiently large. The boundary conditions are

$$
\left.\begin{array}{ll}
V(0, \varphi, t)=K, & \left(0 \leq \varphi<\varphi_{\max }, 0 \leq t \leq T\right), \\
\frac{\partial V}{\partial s}\left(s_{\max }, \varphi, \tau\right)=0, & \left(0 \leq \varphi \leq \varphi_{\max }, 0 \leq \tau \leq T\right), \\
\frac{\partial V}{\partial \varphi}\left(s, \varphi_{\max }, \tau\right)=0, & \left(0 \leq s<s_{\max }, 0 \leq \tau \leq T\right) .
\end{array}\right\}
$$

We use the spectral approach for the discretization of (1)-(3) in the next section.

\section{Discretization Using Spectral Methods}

We discretize the two dimensional problem (1) by using the spectral method. To this end, as a basis, we recall the discretization of a one-dimensional function and then we extend the discretization to the two-dimensional problem by using the tensor product.

To begin with, let us note that the rational approximation of a function $u$, defined on $[-1,1]$, at the Chebyshev points $\xi_{k}, k=0,1, \ldots, N$

$$
u_{N}(\xi):=\frac{\sum_{k=0}^{N} \frac{w_{k}}{\xi-\xi_{k}} u\left(\xi_{k}\right)}{\sum_{k=0}^{N} \frac{w_{k}}{\xi-\xi_{k}}},
$$

where $w_{k}, k=0,1, \ldots, N$ are the barycentric weights defined by $w_{0}:=1 / 2, w_{N}:=(-1)^{N} / 2$, and $w_{k}:=(-1)^{k} ; k=1, \ldots, N-1$. For the rational spectral method, the $m$ th order differentiation matrix associated with the rational interpolant (4) is given by

$$
\begin{aligned}
u_{N}^{(m)}\left(\xi_{k}\right) & =\sum_{k=0}^{N} \frac{d^{m}}{d \xi^{m}}\left(\frac{\sum_{k=0}^{N} \frac{w_{k}}{\xi-\xi_{k}} u\left(\xi_{k}\right)}{\sum_{k=0}^{N} \frac{w_{k}}{\xi-\xi_{k}}}\right) \\
& =\sum_{k=0}^{N} D_{j k}^{(m)} u\left(\xi_{k}\right),
\end{aligned}
$$


Where $D_{j k}^{(m)}$ are the entries of the differentiation matrix of order $m$. The formulas to Construct ${ }^{D_{N}^{(m)}}$ were given by Schneider and Werner in [26] for $m=1$ and $m=2$ and were latter generalized for any order by Tee in [27]. The first and second order differentiation matrices are given by the following formulas

$$
D_{j k}^{(1)}= \begin{cases}\frac{w_{k}}{w_{j}\left(\xi_{j}-\xi_{k}\right)}, & j \neq k, \\ -\sum_{i \neq k} D_{j i}^{(1)}, & j=k,\end{cases}
$$

and

$$
D_{j k}^{(2)}=\left\{\begin{array}{lr}
2 D_{j k}^{(1)}\left(D_{j j}^{(1)}-\frac{1}{\xi_{j}-\xi_{k}}\right), & j \neq k, \\
-\sum_{i \neq k} D_{j i}^{(2)}, & j=k .
\end{array}\right.
$$

The above expressions will be useful in the discretization of the two-dimensional problem (1), as we discuss below. Similarly to the one-dimensional approximation, the rational approximation for a two-dimensional function defined on $[-1,1] \times[-1,1]$ is given by

$$
u_{N}(\xi, \tilde{\xi}):=\frac{\sum_{j=0}^{N} \sum_{k=0}^{\tilde{N}} \frac{w_{j} w_{k}}{\left(\xi-\xi_{j}\right)\left(\tilde{\xi}-\tilde{\xi}_{k}\right)} u\left(\xi_{k}, \tilde{\xi}_{k}\right)}{\sum_{j=0}^{N} \sum_{k=0}^{\tilde{N}} \frac{w_{j} w_{k}}{\left(\xi-\xi_{j}\right)\left(\tilde{\xi}-\tilde{\xi}_{k}\right)}},
$$

where $w_{j}, j=0,1, \ldots, N$ and $w k, j=0,1, \ldots, N$ are the barycentric weights defined by $w_{0}$ $:=1 / 2, w_{N}:=(-1)^{N} / 2$, and $w_{j}:=(-1)^{j} ; j=1, \ldots, N-1$, and $w_{N}:=(-1)^{N} / 2$, and $w_{k}$ $:=(-1)^{k} ; k=1, \ldots, N-1$.

In this paper, we proceed differently to discretize the two-dimensional problem (1). We set up a grid based on Chebyshev points independently in each direction $s$ and $\phi$, called the tensor grid [28-30].

Definition 3.1 Let $P \in \mathrm{R}^{\cdot t \times k}$ and $Q \in \mathrm{R}^{m \times n}$. The tensor product, also called the Kronecker product, of $P$ and $Q$ is the matrix defined by

$$
P \otimes Q:=\left(\begin{array}{ccc}
p_{11} Q & \ldots & p_{1 k} Q \\
\vdots & \ddots & \vdots \\
p_{\ell 1} & \ldots & p_{\ell k} Q
\end{array}\right) \in \mathbb{R}^{\ell m \times k n}
$$


On the tensor grid, the discretization of other derivatives reads

$$
\begin{aligned}
& \frac{\partial V}{\partial s}:=\left(D_{s}^{(1)} \otimes I_{\varphi}\right) V, \\
& \frac{\partial V}{\partial \varphi}:=\left(I_{s} \otimes D_{\varphi}^{(1)}\right) V, \\
& \frac{\partial^{2} V}{\partial s^{2}}:=\left(D_{s}^{(2)} \otimes I_{\varphi}\right) V, \\
& \frac{\partial^{2} V}{\partial \varphi^{2}}:=\left(I_{s} \otimes D_{\varphi}^{(2)}\right) V, \\
& \frac{\partial^{2} V}{\partial s \partial \varphi}:=\left(D_{s}^{(1)} \otimes D_{\varphi}^{(1)}\right) V,
\end{aligned}
$$

where $\otimes$ denotes the Kronecker product, $I_{S}$ and $I_{\phi}$ are the identity matrices in the $s$ and $\phi$ direction, respectively; $\stackrel{D_{s}^{(1,2)}, D_{\varphi}^{(1,2)}}{.}$ are the first and second order differentiation matrices in the corresponding variable.

To discretize (1), we map each domain to the reference interval $[-1,1]$ by the linear transformations

$$
\left.\begin{array}{l}
s=\frac{s_{\max }}{2}(\xi+1) \quad s \in\left[0, s_{\max }\right], \\
\varphi=\frac{\varphi_{\max }}{2}(\xi+1) \quad \varphi \in\left[0, \varphi_{\max }\right] .
\end{array}\right\}
$$

On the tensor grid, (1) becomes

$$
\begin{aligned}
V_{\tau}= & \beta_{1}\left(D_{s}^{(2)} \otimes I_{\varphi}\right) V+\beta_{2}\left(D_{s}^{(1)} \otimes D_{\varphi}^{(1)}\right) V+\beta_{3}\left(I_{s} \otimes D_{\varphi}^{(2)}\right) V \\
& +r\left(D_{s}^{(1)} \otimes I_{\varphi}\right) V+\beta_{4}\left(I_{s} \otimes D_{\varphi}^{(1)}\right) V-r I_{s} \otimes I_{\varphi} V
\end{aligned}
$$

Where 


$$
\begin{aligned}
& \beta_{1}=\frac{1}{2} \sigma^{2} s^{2} I_{s} \otimes \varphi I_{\varphi}, \\
& \beta_{2}=\rho \sigma s I_{s} \otimes \varphi I_{\varphi}, \\
& \beta_{3}=\frac{1}{2} \sigma^{2} \varphi I_{\varphi} \otimes I_{\varphi}, \\
& \beta_{4}=\kappa(\varsigma-\varphi) I_{\varphi} \otimes I_{\varphi} .
\end{aligned}
$$

Now (13) can be written in the form of a global matrix as

$$
V_{\tau}=A V \text {, }
$$

Where

$$
\begin{aligned}
A= & \beta_{1}\left(D_{s}^{(2)} \otimes I_{\varphi}\right)+\beta_{2}\left(D_{s}^{(1)} \otimes D_{\varphi}^{(1)}\right)+\beta_{3}\left(I_{s} \otimes D_{\varphi}^{(2)}\right) \\
& +r\left(D_{s}^{(1)} \otimes I_{\varphi}\right)+\beta_{4}\left(I_{s} \otimes D_{\varphi}^{(1)}\right)-r I_{s} \otimes I_{\varphi} .
\end{aligned}
$$

We solve (14) by the Laplace transform method discussed below.

\section{Application of Laplace Transform to Solve the Semi-discrete Problem}

In this section, we consider the Laplace transform for integrating the parabolic problem (14) with an initial condition $V_{\mathrm{O}}$ and where $A$ is a parabolic operator with its eigenvalues located in the region $\Sigma \delta=\{z \in \mathrm{C}: \mid \arg (z)<\delta, z /=0\}$, for some $\delta \in(0, \pi / 2)$. Furthermore, the resolvent $(z I-A)^{-1}$ of $A$ satisfies

$$
\left\|(z I-A)^{-1}\right\| \leq \frac{C}{1+|z|}, \quad \text { for } z \in \mathbb{C} \backslash \Sigma_{\delta},
$$

for some constant $C>0$ independent of $z$. Note that this implies that the function $V$ admits a holomorphic and bounded extension to a region containing $t \geq 0$, a familiar situation arising, for example, in the context of parabolic problems.

A direct application of the Laplace transform to (14) leads to

$$
(z I-A) \widehat{V}=V_{0},
$$

where $I$ is the identity matrix and and $\widehat{V}^{\prime}$ the Laplace transform of $V(\cdot, t)$ defined by 


$$
\widehat{V}(\cdot, z):=\int_{0}^{\infty} V(\cdot, t) e^{-z t} d t
$$

The inverse is evaluated on a contour $\Gamma$, known as the Bromwich contour, as

$$
V(\cdot, t)=\frac{1}{2 \pi i} \int_{\Gamma} e^{z t} \widehat{V}(\cdot, z) d z, \quad t>0 .
$$

The contour $\Gamma$ is chosen such that it encloses all the singularities of $\widehat{V}^{\prime}(\cdot, z)$.

Our numerical method for inverting the Laplace transform is based on the method developed by Talbot [31] and uses the deformation of the Bromwich contour. The integral is then evaluated using the trapezoidal rule. Talbot's idea was to deform the Bromwich line into a contour which starts and ends in the left half-plane. Such a deformation of the contour is possible by the Cauchy's integral theorem [32]. Cauchy's theorem is applicable, provided that all singularities of the transformed function $\widehat{V}^{\prime}(\cdot, z)$ are contained in the interior of the new contour and that $\left|\widehat{V}^{\prime}(\cdot, z)\right| \rightarrow 0$ as $|z| \rightarrow \infty$ in the half-plane [33]. Such contours are used in $[31,33,34]$, all of which are of the form

$$
z=z(\ell), \quad-\infty<\ell<\infty
$$

with the property that $\operatorname{Re} z \rightarrow-\infty$ as $. e \rightarrow \pm \infty$.

The efficiency of the Talbot approach depends on the choice of the contour, as well as the number of function evaluations in the trapezoidal rule. Simpler contours, such as hyperbolas and parabolas, are proposed in [33,34]. These contours display a better convergence rate than the original cotangent contour used by Talbot. In this paper, we consider the hyperbola as the integration contour defined by

$$
z(\ell):=\widetilde{\mu}(1+\sin (i \ell-\alpha)), \quad \ell \in \mathbb{R},
$$

where the real parameters $\tilde{\mu}>0$ and $\mathrm{o}<\alpha<\pi / 2$ determine the geometry of the contour. The positive parameter $\tilde{\mu}$ controls the width of the contour, while $\alpha$ determines its geometric shape, i.e., the asymptotic angle. On the contour (19) the inversion formula (18) can be rewritten as 


$$
V(\cdot, t)=\frac{1}{2 \pi i} \int_{-\infty}^{\infty} e^{z(\ell) t} \widehat{V}(\cdot, z(\ell)) z^{\prime}(\ell) d \ell,
$$

Where

$$
z^{\prime}(\ell)=\widehat{\mu} i \cos (i \ell-\alpha) .
$$

For $h>0$ such that $l_{k}=k h$, where $k$ is an integer, the trapezoidal rule can then be expressed by

$$
V(\cdot, t) \approx \frac{h}{2 \pi i} \sum_{k=-\infty}^{\infty} e^{z\left(\ell_{k}\right) t} \widehat{V}\left(\cdot, z\left(\ell_{k}\right)\right) z^{\prime}\left(\ell_{k}\right) .
$$

In practice, the infinite sum has to be truncated at a finite integer $M$, in which case one commits a truncation error as discussed below. Note that, because of the symmetry of the contour (19), (21) can be rewritten as

$$
V_{M}(\cdot, t) \approx \operatorname{Re}\left\{\frac{h}{\pi i} \sum_{k=0}^{M} \circ e^{z\left(\ell_{k}\right) t} \widehat{V}\left(\cdot, z\left(\ell_{k}\right)\right) z^{\prime}\left(\ell_{k}\right)\right\},
$$

where 'o' indicates that the first term is divided by 2 . The benefit of using (22) is that it reduces by half the summation (21) and subsequently the number of linear systems to be evaluated in (16).

In the following subsections, we analyze the overall error that occurs during the approximation of the solution using our method for integration in time.

\subsection{Analysis of the Error}

In this section, we analyze the error associated with the use of the Laplace transform for integration in the time direction. To this end, first we note that the application of the trapezoidal rule (21) to the unbounded integral (20) introduces discretization error. Second, a truncation of the infinite series (21) at a finite integer $M$ (for practical implementation) produces a truncation error as one would expect. Furthermore, since the evaluation of (21) is done in a floating point environment, a roundoff error is also introduced at each evaluation there. This roundoff error may increase dramatically and affects the accuracy of numerical solution due to the exponential factor involved in (21), as we will discuss below. The error due to the use of the Laplace transform is therefore the sum of the discretization, truncation and conditioning errors. 


\subsubsection{Discretization Error}

The discretization error is the difference between the continuous formula (20) and the corresponding trapezoidal formula (21), i.e.,

$$
\begin{aligned}
E_{d}= & \frac{1}{2 \pi i} \int_{-\infty}^{\infty} e^{z(\ell) t} \widehat{V}(\cdot, z(\ell)) z^{\prime}(\ell) d \ell \\
& -\frac{h}{2 \pi i} \sum_{k=-\infty}^{\infty} e^{z\left(\ell_{k}\right) t} \widehat{V}\left(\cdot, z\left(\ell_{k}\right)\right) z^{\prime}\left(\ell_{k}\right) .
\end{aligned}
$$

To estimate the discrete error (23), the idea is to use the contour integral to represent $E_{d}$. This approach based on the Cauchy's Residue theorem in complex analysis was originally developed by Martensen for an analytic function $f(t)$ defined on $(-\infty, \infty)$ [35]. In that paper, the author showed that for an analytic function, the trapezoidal rule (21) converges exponentially, as illustrated in the following theorem.

Theorem 4.1 Let $f: \mathrm{R} \rightarrow \mathrm{R}$ be an analytic function. Then there exists a strip $\mathrm{R} \times(-d, d)$ in the complex plane with $d>0$ such that $f$ can be extended to a complex analytic function $f: \mathrm{R} \times(-d, d) \rightarrow \mathrm{C}$. Furthermore, the error for the trapezoidal rule indicated in (23) is given by

$$
E_{d}=\operatorname{Re}\left\{\int_{-\infty+i \theta}^{\infty+i \theta}\left(1-i \cot \frac{\pi z}{h}\right) f(z) d z\right\},
$$

where $0<\theta<d$. Moreover, it is bounded by

$$
\left|E_{d}\right| \leq \frac{2 C}{e^{2 \pi d / h}-1},
$$

where $C$ is a constant such that

$$
\int_{-\infty+i \theta}^{\infty+i \theta}|f(z)| d z \leq C .
$$

Proof See [35].

From the above theorem, clearly,

$$
\left|E_{d}\right| \leq \frac{2 e^{-2 \pi d / h} C}{1-e^{-2 \pi d / h}} \rightarrow 0 \quad \text { as } h \rightarrow 0 .
$$


For further discussion on the convergence properties of the trapezoidal rule, we refer the readers to [35].

\subsubsection{Truncation Error}

The truncation error is the error made by ignoring the remaining terms in (21) after truncating the series at a finite number $M$, and is given by

$$
\begin{aligned}
E_{t} & =\frac{h}{2 \pi i} \sum_{k=-\infty}^{\infty} e^{z\left(\ell_{k}\right) t} \widehat{V}\left(\cdot, z\left(\ell_{k}\right)\right) z^{\prime}\left(\ell_{k}\right)-\frac{h}{2 \pi i} \sum_{k=-\infty}^{\infty} e^{z\left(\ell_{k}\right) t} \widehat{V}\left(\cdot, z\left(\ell_{k}\right)\right) z^{\prime}\left(\ell_{k}\right) \\
& =\frac{h}{2 \pi i}\left(\sum_{k=-\infty}^{N-1} e^{z\left(\ell_{k}\right) t} \widehat{V}\left(\cdot, z\left(\ell_{k}\right)\right) z^{\prime}\left(\ell_{k}\right)+\sum_{k=N+1}^{\infty} e^{z\left(\ell_{k}\right) t} \widehat{V}\left(\cdot, z\left(\ell_{k}\right)\right) z^{\prime}\left(\ell_{k}\right)\right) .
\end{aligned}
$$

Note that we have $\widehat{\widehat{V}\left(\cdot, z\left(\ell_{k}\right)\right)}=\widehat{V}\left(\cdot, \overline{z\left(\ell_{k}\right)}\right)$ and $\overline{z\left(\ell_{k}\right)}=z\left(\ell_{-k}\right)$; and since the contour is symmetric, (25) becomes

$$
E_{t}=\frac{h}{\pi i} \sum_{k=N+1}^{\infty} e^{z\left(\ell_{k}\right) t} \widehat{V}\left(z\left(\ell_{k}\right)\right) z^{\prime}\left(\ell_{k}\right)
$$

Because of the exponential factor $e^{z(l k) t}$, the terms in the sum decrease exponentially as $k \rightarrow$ $\infty$, and therefore in this case one commits only an exponentially small error whose contribution therefore can be neglected.

\subsubsection{Conditioning Error}

To study the conditioning error in the application of the Laplace transform, re- call that in (21), the approximation $\tilde{f}(t)$ requires the evaluation of the transformed $F(z k)=F\left(z\left(l_{k}\right)\right)$, for $k=-M,-M+1, \ldots, M-1, M$. In reality, these evaluations are affected by round-off errors which means that the actual approximation that takes place is

$$
\tilde{F}\left(z_{k}\right)=\widetilde{V}\left(\cdot, z_{k}\right)+\rho_{k}, \quad k=-M,-M+1, \ldots, M-1, M,
$$


where $\rho_{k}>0$ is a small value such that $\left|\rho_{k}\right| \leq \epsilon$, with $\epsilon$ given by the machine precision. As a result, (21) implies

$$
\begin{aligned}
\check{f}_{M}(t) & =\frac{h}{2 \pi i} \sum_{k=-M}^{M} e^{z_{k} t}\left(\widetilde{V}\left(\cdot, z_{k}\right)+\rho_{k}\right) z_{k}^{\prime} \\
& =V_{M}(t)+\frac{h}{2 \pi i} \sum_{k=-M}^{M} e^{z_{k} t} \rho_{k} z_{k}^{\prime} .
\end{aligned}
$$

We set

$$
f_{\rho}(t)=\frac{h}{2 \pi i} \sum_{k=-M}^{M} e^{z_{k} t} \rho_{k} z_{k}^{\prime} .
$$

Before we proceed, we state the following lemma [34]. Let

$$
L(x)=1+\left|\ln \left(1-e^{-x}\right)\right|, \quad x>0 .
$$

The function $L$ is such that $L(x) \rightarrow 1$ as $x \rightarrow \infty$, and $L(x) \sim|\ln x|$ as $x \rightarrow 0^{+}$. This function $L$ has the property stated in the following lemma.

Lemma 4.1 The function L defined above satisfies the following inequality

$$
\int_{0}^{\infty} e^{-\gamma \cosh x} d x \leq L(\gamma), \quad \gamma>0 .
$$

Proof Consider the change of variable $\varphi=\cosh x-1$ so that

$$
\int_{0}^{\infty} e^{-\gamma \cosh x} d x=e^{-\gamma} \int_{0}^{\infty} \frac{e^{-\gamma \varphi}}{\sqrt{\varphi(2+\varphi)}} d \varphi .
$$

Set $a=\sinh ^{2}(1 / 2)$, so that

$$
\int_{0}^{a} \frac{d \varphi}{\sqrt{\varphi(2+\varphi)}}=1 \quad \text { and } \quad \frac{1}{\sqrt{(k+a)(2+k+a)}} \leq \frac{1}{k+1}, \quad k \geq 0 .
$$

Thus

$$
\begin{aligned}
e^{-\gamma} \int_{0}^{\infty} \frac{e^{-\gamma \varphi}}{\sqrt{\varphi(2+\varphi)}} d \varphi & \leq e^{-\gamma}\left(1+\sum_{k=0}^{\infty} e^{-\gamma k} \int_{a+k}^{a+k+1} \frac{d \varphi}{\sqrt{\varphi(2+\varphi)}}\right) \\
& \leq e^{-\gamma}\left(1+\sum_{k=0}^{\infty} \frac{e^{-\gamma k}}{k+1}\right)=e^{-\gamma}+L(\gamma)-1,
\end{aligned}
$$

Which completes the proof. 
To see how the conditioning error affects the numerical results, we need to estimate $\left\|f_{\rho}(t)\right\|$. To this end, note that

$$
\begin{aligned}
\left\|f_{\rho}(t)\right\| & =\left\|\frac{h}{2 \pi i} \sum_{k=-M}^{M} e^{z_{k} t} \rho_{k} z_{k}^{\prime}\right\| \\
& =\left\|\frac{\tilde{\mu} e^{\widetilde{\mu} t}}{2 \pi} h \sum_{k=-M}^{M} e^{\tilde{\mu} \sin \left(i \ell_{k}-\alpha\right) t} \rho_{k} \cos (i \ell-\alpha)\right\|, \quad \alpha \in(\pi / 4, \pi / 2), \\
& \leq \frac{\tilde{\mu} e^{\tilde{\mu} t}}{2 \pi} h \sum_{k=-M}^{M}\left\|e^{\tilde{\mu} \sin \left(i \ell_{k}-\alpha\right) t} \rho_{k} \cos (i \ell-\alpha)\right\| .
\end{aligned}
$$

The following two inequalities are proved in [34], thus here we only state them in our context. One of them is

$$
\left\|e^{\tilde{\mu} t \sin (i \ell-\alpha)} \rho_{k} \cos (i \ell-\alpha)\right\| \leq \rho e^{-\widetilde{\mu} t \sin \alpha \cosh \ell} \cos (i \ell-\alpha),
$$

whereas the other one is

$$
e^{-\widetilde{\mu} t \sin \alpha \cosh \ell} \cos (i \ell-\alpha) \leq \frac{1}{e \widetilde{\mu} \varrho \sin \alpha t} e^{-(1-\varrho) \widetilde{\mu} t \sin \alpha \cosh \ell}, \quad \varrho \in(0,1) .
$$

From (27) and (28), we deduce that

$$
\begin{aligned}
\left\|f_{\rho}(t)\right\| & \leq \frac{e^{\tilde{\mu} t} \rho}{2 \pi e \varrho \sin \alpha} \frac{h}{t} \sum_{k=-M}^{M} e^{-(1-\varrho) \tilde{\mu} t \sin \alpha \cosh \ell_{k}}, \\
& \leq \frac{e^{\tilde{\mu} t} \rho}{\pi e \varrho \sin \alpha} \frac{h}{t} \sum_{k=0}^{\infty} e^{-(1-\varrho) \tilde{\mu} t \sin \alpha \cosh \ell_{k}}, \\
& \leq \frac{e^{\widetilde{\mu} t}}{\pi e \varrho \sin \alpha} \frac{\rho}{t} \int_{0}^{\infty} e^{-(1-\varrho) \tilde{\mu} t \sin \alpha \cosh \ell} .
\end{aligned}
$$

Using Lemma 4.1, we obtain

$$
\left\|f_{\rho}(t)\right\| \leq \frac{e^{\tilde{\mu} t}}{\pi e \varrho \sin \alpha} \frac{\rho}{t}[L((1-\varrho) \tilde{\mu} t \sin \alpha)] .
$$

We note that (30) is independent of $\widehat{V}(\cdot, z)$ and propagates moderately with respect to $\tilde{\mu} t$. . 
In summary, (24), the argument mentioned just after (26) and (30) imply that the total error is fully controllable as long as we choose the optimal values of the associated parameters. The derivation of these parameters is described below.

\subsection{Derivation of the Optimal Contour Parameters}

In this subsection, we derive optimal parameters of the contour (19) on which the integral is to be evaluated. To this end, we note that the computational effort in the evaluation of $V M$ $(\cdot, t)$ in $(21)$ comes from the evaluation of the Laplace transform $\widehat{V}(\cdot, z)$ in (16) for each $z\left(l_{k}\right)$. Furthermore, we note that this evaluation of $\widehat{V}(\cdot, t)$ is independent of time $t$ and thus can be carried out once, and subsequently use the same evaluation to approximate $V M(\cdot, t)$ at a different time level over an interval $\left[t_{0}, \Lambda t_{\mathrm{O}}\right]$ for an integer $\Lambda$. Note also that various methods for finding an optimal contour were developed in [33, 34]. In [33], the following convergence estimate for the family of hyperbolic contours were derived

$$
E_{d}=\mathcal{O}\left(e^{-2 \pi(\pi / 2-\alpha) / h}\right), \quad E_{-d}=\mathcal{O}\left(e^{\tilde{\mu} t-2 \pi \alpha / h}\right), \quad h \rightarrow 0 .
$$

Therefore, the total discretization error on the strip $(-d, d)$, denoted by $E r$, is given by

$$
E_{r}:=E_{d}+E_{-d} .
$$

Moreover, the truncation error that we found satisfies

$$
E_{t}=\mathcal{O}\left(e^{\widetilde{\mu} t(1-\sin \alpha \cosh (h M))}\right), \quad M \rightarrow \infty .
$$

To obtain an optimal contour parameter, we argue as follows: first note that only the second equation in (31) and (32) are time dependent. On the one hand, the erro $r E_{t}$ decreases when $t$ increases and thus is maximum at $t_{0}$. To see this, we consider $\alpha \in(\pi / 4, \pi / 2)$, which implies that the inequality $1^{\sqrt{2}}<\sin \alpha<1$ holds. For $M \rightarrow \infty$, multiplication of both sides of the inequality by $\cosh (h M)$ yields

$$
\frac{\cosh (h M)}{\sqrt{2}}<\sin \alpha \cosh (h M)<\cosh (h M) .
$$

Since $\cosh (h M) / \sqrt{2}>1$ for sufficiently large $M$, we get

$$
1-\sin \alpha \cosh (h M) \leq 0, \quad \text { for a fixed } h \neq 0 \text { and } M \rightarrow \infty .
$$


On the other hand, the discretization error $E_{-} d$ increases with $t$, and so is maximum at $t=$ $\Lambda t_{\mathrm{O}}$.

To estimate the optimal parameters of the contour on $\left[t_{0}, \Lambda t_{0}\right]$, an asymptotic balance of the three errors at their maximum, i.e., $E_{t}, E_{d}, E_{-d}$ is required. To this end, we set

$$
-2 \pi(\pi / 2-\alpha) / h=\widetilde{\mu} \Lambda t_{0}-2 \pi \alpha / h=\widetilde{\mu} t_{0}(1-\sin \alpha \cosh (h M)) .
$$

We solve these equations for $\tilde{\mu}$ and $h$. From the first equation, we have

$$
\tilde{\mu} h=\frac{4 \alpha \pi-\pi^{2}}{\Lambda t_{0}} .
$$

The last equation in (33), together with (34), yields

$$
\cosh (h M)=\frac{(\pi-2 \alpha) \Lambda-\pi+4 \alpha}{(4 \alpha-\pi) \sin \alpha} .
$$

From this it follows that

$$
A(\alpha)=h M=\cosh ^{-1}\left(\frac{(\pi-2 \alpha) \Lambda-\pi+4 \alpha}{(4 \alpha-\pi) \sin \alpha}\right) .
$$

Therefore, we obtain

$$
h=\frac{A(\alpha)}{M}
$$

and

$$
\tilde{\mu}=\frac{4 \alpha \pi-\pi^{2}}{h \Lambda t_{0}}=\frac{4 \alpha \pi-\pi^{2}}{A(\alpha)} \frac{M}{\Lambda t_{0}} .
$$

The contour parameters (37) and (36) are fixed and time independent. As a result, the corresponding contour (19) is also fixed over the interval $\left[t_{0}, \Lambda t_{\mathrm{O}}\right]$. From the parameters derived above, the error is

$$
E_{M}=\mathcal{O}\left(e^{-B(\alpha) M}\right),
$$

where

$$
B(\alpha)=\frac{\pi^{2}-2 \pi \alpha}{\cosh ^{-1}\left(\frac{(\pi-2 \alpha) \Lambda+4 \alpha-\pi}{(4 \alpha-\pi) \sin \alpha}\right)} .
$$


Table 1 Optimal parameters of the contour (19) for $\Lambda=50$

\begin{tabular}{lllll}
\hline$\Lambda$ & $\alpha$ & $A(\alpha)$ & $\mu \Lambda t_{0} / M$ & $B(\alpha)$ \\
\hline 50 & 0.9381 & 5.5582 & 0.3452 & 0.7152 \\
\hline
\end{tabular}

Table 2 Values of the European put option associated with the Heston's volatility model for $\varphi=0.0625$

\begin{tabular}{llll}
\hline $\mathrm{s}$ & Exact values [12] & ILT & Error \\
\hline 8 & 2.0000 & 2.0984 & 0.0984 \\
9 & 1.1080 & 1.1523 & 0.0443 \\
10 & 0.5316 & 0.5422 & 0.0106 \\
11 & 0.2261 & 0.1502 & 0.0759 \\
12 & 0.0907 & 0.0788 & 0.0119 \\
\hline
\end{tabular}

Table 3 Values of the European put option associated with the Heston's volatility model for $\varphi=0.25$

\begin{tabular}{rlll}
\hline $\mathrm{s}$ & Exact values [12] & ILT & Error \\
\hline 8 & 2.0733 & 2.0930 & 0.0197 \\
9 & 1.3290 & 1.3460 & 0.0170 \\
10 & 0.7992 & 0.6974 & 0.1018 \\
11 & 0.4536 & 0.4699 & 0.0163 \\
12 & 0.2502 & 0.2611 & 0.0109 \\
\hline
\end{tabular}

The optimal error is obtained when $B(\alpha)$ attains its maximum for each value of $\Lambda$. In our computation, we choose an $\Lambda=50$ and obtained optimal parameters as listed in Table 1 .

Below we discuss the numerical results.

\section{Numerical Results}

In this section, we compute the European put option prices for the Heston's model (14) with boundary conditions (3) using the spectral method in space and the contour integral method in the time directions. The number of mesh discretization points in the space (asset) and the volatility space directions are 25 and 15 , respectively. The other parameter values used in the simulation are $K=10 ; \sigma=0.9 ; r=0.1 ; \rho=0.1 ; \varsigma=0.16 ; T=0.25, s \max =20, \phi \max =1$. The prices are presented in Tables 2 and 3 for asset values $s=8 ; 9 ; 10 ; 11 ; 12$ and for the variance values $\phi=0.0625$ and $\phi=0.25$. In the first column, we have the values of the asset, the second column contains the exact values obtained in [12], the third column contains the values obtained from our inverse Laplace transform (ILT) approach, and the last column represents the estimated error.

From Tables 2 and 3, we observe that the direct application of the spectral method combined with the ILT method produces results which are second order accurate. 


\section{Concluding Remarks and Scope for Future Research}

We proposed the spectral method combined with the Laplace transform method for pricing European options using Heston's stochastic volatility model. In this approach, a direct spectral method is applied for discretization in space. To approximate the derivatives in both $s$ and $v$ directions, we set up a grid based on Chebyshev points independently in both directions and then considered a new grid, which is the tensor product of these two grids. Our computational results show that the proposed method is second order accurate.

Currently, we are investigating the possibility of using the multi-domain decomposition method in space to further improve the accuracy. This may further be augmented with the use of a higher order time integration technique.

\section{Acknowledgements}

The two authors, Ngounda and Pindza, acknowledge the Agence National des Bourses du Gabon for the financial support. Patidar's research was supported by the South African National Research Foundation. Furthermore, we acknowledge the anonyms referees for their valuable comments and suggestions. 


\section{References}

Hull, J.C., White, A.: The pricing of options on assets with stochastic volatility. J. Finance 42(2), 281-300 (1987)

Merton, R.C.: Option pricing when underlying stock returns are discontinuous. J. Financ. Econ. 3, 125-144 (1975)

Heston, S.L.: A closed-form solution for options with stochastic volatility with applications to bond and currency options. Rev. Financ. Stud. 6(2), 327-343 (1993)

In't Hout, K.J., Foulon, S.: ADI finite difference schemes for option pricing in the Heston model with correlation. Int. J. Numer. Anal. Model. 7(2), 303-320 (2010)

Douglas, J., Rachford, H.H.: On the numerical solution of heat conduction problems in two and three space variables. Trans. Am. Math. Soc. 82, 421-439 (1956)

McKee, S., Wall, D.P., Wilson, S.K.: An alternating direction implicit scheme for parabolic equations with mixed derivative and convective terms. J. Comp. Physiol. 126(1), 64-76 (1996)

Craig, I.J.D., Sneyd, A.D.: An alternating-direction implicit scheme for parabolic equations with mixed derivatives. Comput. Math. Appl. 16, 341-350 (1988)

In't Hout, K.J., Welfert, B.D.: Unconditional stability of second-order ADI schemes applied to multi- dimensional diffusion equations with mixed derivative. Appl. Numer. Math. 59(3-4), 677-692 (2009)

Hundsdorfer, W.: Accuracy and stability of splitting with stabilizing corrections. Appl. Numer. Math.

42, 213-233 (2002)

Hundsdorfer, W., Verwer, J.G.: Numerical Solution of Time-Dependent AdvectionDiffusion- Reaction Equations. Springer, Berlin (2003)

Haentjens, T.: ADI finite difference discretization of the Heston-Hull-White PDE. In: AIP Con- ference Proceedings, ICNAAM 2010: International Conference of Numerical Analysis and Applied Mathematics 2010, vol. 1281, pp. 1995-1999 (2010)

Ikonen, S., Toivanen, J.: Operator splitting methods for pricing American options under stochastic volatility. Numer. Math. 113(2), 299-324 (2009)

Oosterlee, C.W.: On multigrid for linear complementarity problems with application to American- style options. Electron. Trans. Numer. Anal. 15, 165-185 (2003)

Zvan, R., Forsyth, P.A., Vetzal, K.R.: Penalty methods for American option with stochastic volatility. J. Comput. Appl. Math. 91(2), 199-218 (1998)

Zhu, S.P., Chen, W.T.: A new analytical approximation for European puts with stochastic volatility. Appl. Math. Lett. 23, 687-692 (2010)

In't Hout, K.J., Weideman, J.A.C.: Appraisal of a contour integral method for the BlackScholes and Heston equations. SIAM J. Sci. Comput. 33, 763-785 (2010)

Albrecher, H., Mayer, P., Schoutens, W., Tistaert, J.: The little Heston trap. Wilmott Mag. January, 83-92 (2007) 
Carr, P., Madan, D.: Option valuation using the Fast Fourier Transform. J. Comput. Finance 2(4), 61-73 (1999)

Clarke, N., Parrott, K.: Multigrid for American option pricing with stochastic volatility. Appl. Math. Finance 6, 177-195 (1999)

Gatheral, J.: The Volatility Surface: A Practioner's Guide. Wiley Finance, New York (2006)

Grzelak, L.A., Oosterlee, C.W.: On the Heston model with stochastic interest rates. SIAM J. Financ. Math. 2, 255-286 (2011)

Kahl, C., Jäckel, P.: Not-so-complex logarithms in the Heston model. Wilmott Mag. September, 94- 103 (2005)

Kim, J., Kim, B., Moon, K.-S., Wee, I.-S.: Valuation of power options under Heston's stochastic volatility model. J. Econ. Dyn. Control 36(11), 1796-1813 (2012)

Langrock, R., MacDonald, I.L., Zucchini, W.: Some nonstandard stochastic volatility models and their estimation using structured hidden Markov models. J. Empir. Finance 19(1), 147-161 (2012)

Laurent, S., Rombouts, J.V.K., Violante, F.: On loss functions and ranking forecasting performances of multivariate volatility models. J. Economet. 173(1), 1-10 (2013)

Schneider, C., Werner, W.: Some new aspect of rational interpolation. Math. Comput. 47(175), 285- 299 (1986)

Tee, W.: A rational collocation with adaptively transformed Chebyshev grid points. SIAM J. Sci. Comput. 28(5), 8-1811 (2006)

Heinrichs, W.: Improved condition number of spectral methods. Math. Comput. 53(187), 103-119 (1989)

Julien, K., Watson, M.: Efficient multi-dimensional solution of PDEs using Chebyshev spectral meth- ods. J. Comp. Physiol. 228(5), 1480-1503 (2009)

Trefethen, L.N.: Spectral Method in MATLAB. SIAM, Philadelphia (2000)

Talbot, A.: The accurate numerical inversion of Laplace transforms. IMA J. Appl. Math. 23(1), 97- 120 (1979)

Spiegel, M.R.: Theory and Problems of Laplace Transforms. McGraw-Hill, New York (1965)

Weideman, J.A.C., Trefethen, L.N.: Parabolic and hyperbolic contours for computing the Bromwich integral. Math. Comput. 76(259), 1341-1356 (2007)

López-Fernández, M., Palencia, C.: On the numerical inversion of the Laplace transform of certain holomorphic mappings. Appl. Numer. Math. 51(2-3), 289-303 (2004)

Martensen, V.E.: Zur numerischen Auswertung uneigentlicher Integrale. Z. Angew. Math. Mech. 48, T83-T85 (1968) 